"This document is the Accepted Manuscript version of a Published Work that appeared in final form in Langmuir, copyright (c) American Chemical Society after peer review and technical editing by the publisher.

To access the final edited and published work see [http://dx.doi.org/10.1021/acs.langmuir.7b00429]."

\title{
Stability of clay particle-coated microbubbles in alkanes against dissolution induced by heating
}

\author{
Kanvara Achakulwisut, ${ }^{1 \dagger}$ Chak Tam,,${ }^{1 \dagger}$ Axel Huerre, ${ }^{1}$ Rafaella Sammouti, ${ }^{1}$ \\ Bernard P. Binks ${ }^{2}$ and Valeria Garbin ${ }^{1 *}$ \\ ${ }^{1}$ Department of Chemical Engineering, Imperial College London, Exhibition Road, \\ London SW7 2AZ, UK \\ ${ }^{2}$ School of Mathematics and Physical Sciences, University of Hull, Hull HU6 7RX, UK \\ *Author for correspondence: v.garbin@imperial.ac.uk
}

\begin{abstract}
We investigated the dissolution and morphological dynamics of air bubbles in alkanes stabilized by fluorinated colloidal clay particles when subjected to temperature changes. A quasi-steady model for bubble dissolution with time-dependent temperature reveals that increasing the temperature enhances the bubble dissolution rate in alkanes, opposite to the behavior in water, due to the differing trends in gas solubility. Experimental results for uncoated air bubbles in decane and hexadecane confirm this prediction. Clay-coated bubbles in decane and hexadecane are shown to be stable in air-saturated oil at constant temperature, where dissolution is driven mainly by the Laplace pressure. When the temperature increases from ambient, the particle-coated bubbles are prone to dissolution as the oil phase becomes under-saturated. The interfacial layer of particles is observed to undergo buckling and crumpling, without shedding of clay particles. Increasing the concentration of particles is shown to enhance the bubble stability by providing a higher resistance to dissolution and buckling. When subjected to complex temperature cycles, the claycoated bubbles can remain stable in conditions for which uncoated bubbles dissolve completely. These results underpin the design of ultra-stable oil foams stabilized by solid particles with improved shelf life under changing environmental conditions.
\end{abstract}

\footnotetext{
${ }^{\dagger}$ These authors contributed equally
} 


\section{Introduction}

Foams used in the food ${ }^{1}$, pharmaceutical, and cosmetic industries are typically based on an aqueous continuous phase. Oil foams are less common but can be problematic, for instance in lubricating oils when used in engines or gearboxes ${ }^{2}$. Because foams are thermodynamically unstable, they tend to age quickly and are destabilized by a combination of liquid drainage, gas disproportionation and coalescence ${ }^{3}$. Foam stability can be achieved by utilizing surfactants, proteins, or colloidal particles. Particle-stabilized bubbles can be much more stable than surfactant- or protein-stabilized bubbles, and the stability can last up to several months compared to only a few hours with proteins ${ }^{1}$.

Particles provide stabilization through adsorption onto the gas-liquid interface, in analogy to surfactant molecules. However, particle adsorption can often be considered to be irreversible, as the free energy of desorption, $\Delta F=\pi \gamma a^{2}(1-|\cos \theta|)$ can be of the order of $10^{3}-10^{6} k_{B} T$, much higher than that of surfactants ${ }^{3,4,5}$. Here, $\gamma$ is the air-liquid surface tension, $a$ is the radius of the particles, assumed to be spherical, $\theta$ is the contact angle the particle makes with the air-liquid interface, and $k_{B} T$ the thermal energy. In addition, particles can form a densely packed layer on the interface, which acts as a strong colloidal armor ${ }^{6}$ that can reduce gas permeation and prevent coalescence. For aqueous foams, it is well understood that parameters such as particle shape, surface charge and bulk concentration also influence the performance of colloidal particles in foam stabilization. A higher bulk concentration of particles has been shown to provide greater stability ${ }^{6,7,8}$, because an increased number of particles adsorb onto the interface, consequently resulting in a denser packing of the particles. This dense packing acts as a shield preventing bubble coalescence and disproportionation.

Oil foams stabilized solely by particles were reported relatively recently ${ }^{9,10,11,12}$. Since the surface tension of the oil-air interface is much lower than that of the air-water interface, particles of lower surface energy than conventional hydrocarbon-coated ones are required for adsorption. The first studies utilized either micron-sized oligotetrafluoroethylene ${ }^{9}$ or polytetrafluoroethylene particles ${ }^{10}$. Stable oil foams were formed for oils with surface tension in the range $30-45 \mathrm{mN} \mathrm{m}^{-1}$, and contact angles ranging between $40^{\circ}$ to $90^{\circ}$ (measured through the oil phase). Increasing the bulk concentration of particles led to more adsorption on the air bubbles, resulting in improved foamability and stability, in keeping with observations for aqueous foams. Studies of the effect of the contact angle using fluorinated silica nanoparticles have confirmed that oil foams are only formed for low values of the cosine of the contact angle ${ }^{11}$. Recently, fluorinated clay platelet particles were also shown to stabilize a range of oil foams ${ }^{12}$. The oleophobicity of the particles was controlled by varying the amount of perfluoroalkyl phosphate diethanolamine salt coating on the particles. Foams were 
formed for oils with sufficiently high surface tension $\left(\gamma>22 \mathrm{mN} \mathrm{m}^{-1}\right)$, including decane and hexadecane.

The effect of changes in temperature on the lifetime and durability of particle-stabilized foams has received limited attention so far, despite its importance for storage conditions of food and personal care products, and for oilfield applications, where foams need to remain stable at high temperature and pressure. A study on the effect of high temperature on surfactant-stabilized aqueous foams for enhanced oil recovery ${ }^{13}$ has shown that foam stability is reduced. A comparison between the use of surfactant only (sodium dodecyl sulfate, SDS), and surfactant in combination with particles (partially hydrophobic silica nanoparticles) revealed that the addition of nanoparticles prevents foam collapse at high temperatures. The gas transport mechanisms occurring at the microscale and at the single bubble level were investigated recently for bubbles in water stabilized by polystyrene microspheres ${ }^{14}$. It was found that a reduction in temperature enhances the dissolution of particle-stabilized air bubbles in water, which otherwise remain stable at fixed temperature. It was shown that the enhanced bubble dissolution is due to the increase in gas solubility in water with decreasing temperature, which causes the continuous phase to become under-saturated and hence drives bubble dissolution. To date, the effect of changes in temperature on the stability of particle-coated bubbles in oil has not been investigated.

In this paper, we study the effect of an increase in temperature on the stability of microbubbles stabilized by fluorinated clay particles in alkanes. We compare results for clay-coated bubbles with those for uncoated bubbles to establish the effect of the particle coating. A model for uncoated-bubble dissolution in a time-dependent temperature field ${ }^{14}$ is used to guide the interpretation of the experimental results. We explore the effect of alkane chain length, bulk concentration of particles, rate of change of temperature and complex heating-cooling cycles on bubble stability, with the goal of identifying conditions for stability in varying temperature conditions.

\section{Theory}

Bubble dissolution with time-dependent temperature. We first review the theory of bubble dissolution at constant temperature ${ }^{15,16,17}$ and with time-dependent temperature ${ }^{14}$. The dissolution of gases in liquids is governed, at low concentration, by Henry's law which states that the equilibrium concentration of dissolved gas in the liquid is proportional to the partial pressure of gas in contact with the liquid ${ }^{17}$ :

$$
c_{s}=k_{H} M P_{g}
$$


where $c_{S}$ is the saturation concentration of dissolved gas at the liquid-gas interface, $P_{g}$ is the partial pressure of the gas and $M$ is the molecular weight of the gas. Henry's constant, $k_{H}$, depends on the nature of the gas and the liquid and on the temperature, T. Epstein and Plesset ${ }^{15}$ obtained an equation for the rate of change of the radius of a bubble, $\dot{R}$, as a function of the saturation ratio $f=c / c_{s}$, where $c$ is the concentration of dissolved gas in the liquid:

$$
\dot{R}=-D k_{H} R_{g} T(1-f)\left(\frac{1}{R}+\frac{1}{\sqrt{\pi D t}}\right)
$$

Here $D$ is the diffusion coefficient of the gas in the liquid and $R_{g}$ is the universal gas constant. It can be seen that in an under-saturated solution $(f<1)$, a bubble shrinks, whereas in a super-saturated solution $(f>1)$, the gas will diffuse from the bulk liquid into the bubble leading to bubble growth.

For bubbles of sufficiently small radius $R$, the gas pressure inside the bubble can be significantly higher than the ambient pressure, due to the Laplace pressure $\Delta p=\frac{2 \gamma}{R}$ such that

$$
P_{g}=P_{0}+\frac{2 \gamma}{R}
$$

where $\gamma$ is the interfacial tension between the gas and the liquid. The concentration of dissolved gas at the bubble interface is therefore higher than the concentration of dissolved gas in the bulk liquid. The rate of change in bubble radius can be modified to account for this effect ${ }^{16}$ :

$$
\dot{R}=-D k_{H} R_{g} T\left(1-f+\frac{2 \gamma / R}{P_{0}}\right)\left(\frac{1}{R}+\frac{1}{\sqrt{\pi D t}}\right)\left(1+\frac{2}{3} \frac{2 \gamma / R}{P_{0}}\right)^{-1} \text {. }
$$

Equation 4 shows that, for sufficiently small bubble radius that the Laplace pressure is significant ${ }^{14}$, the direction of gas diffusion is also dependent on the ratio between the Laplace pressure and the ambient pressure. Small bubbles with sufficiently large Laplace pressure will dissolve even in a supersaturated solution $(f>1)$, provided that $f<1+\frac{2 \gamma / R}{P_{0}}$.

Changes in temperature can influence the dynamics of bubble dissolution. Equation 4 was modified, in the quasi-steady limit, to account for a time-dependent temperature ${ }^{14}$ :

$$
\begin{aligned}
\dot{R}= & -D k_{H} R_{g} T\left(1-f+\frac{2 \gamma / R}{P_{0}}\right)\left(\frac{1}{R}+\frac{1}{\sqrt{\pi D t}}\right)\left(1+\frac{2}{3} \frac{2 \gamma / R}{P_{0}}\right)^{-1}+ \\
& +\dot{T}\left(P_{0} R+2 \gamma-2 T \frac{d \gamma}{d T}\right)\left(3 T\left(P_{0}+\frac{2}{3} \frac{2 \gamma}{R}\right)\right)^{-1} .
\end{aligned}
$$

The diffusion coefficient $D$, Henry's constant, $k_{H}$, and the surface tension, $\gamma$, all depend on temperature. For air bubbles in water, it was found that the rate of dissolution increases upon a 
decrease in temperature ${ }^{14}$. It was shown that the increase of Henry's constant with decreasing temperature causes an increase in the saturation concentration, therefore making the liquid undersaturated and enhancing bubble dissolution. The other temperature-dependent parameters were shown to play a comparatively minor role. Experiments at constant temperature in which a saturated solution was exchanged with an under-saturated solution showed the same enhancement in bubble dissolution and therefore confirmed the proposed mechanism ${ }^{14}$.

Temperature dependence of physicochemical properties of alkanes. We now turn our attention to bubble dissolution in alkanes. Equation 5 will be used, together with a set of equations for the temperature dependence of the physicochemical properties of alkanes. The modified Stokes-Einstein relation $^{18}$,

$$
\frac{D \eta}{T}=\text { constant }
$$

gives the temperature dependence of the diffusion coefficient, $D$, through the temperature dependence of the dynamic viscosity, $\eta$. The following correlations give the temperature dependence of the physicochemical properties of alkanes ${ }^{19,20,21,22,23,24}$ :

$$
\begin{aligned}
& \eta(T)=a_{3} T^{3}+a_{2} T^{2}+a_{1} T+a_{0}, \\
& \gamma(T)=b_{3} T^{3}+b_{2} T^{2}+b_{1} T+b_{0}, \\
& k_{H}(T)=c_{3} T^{3}+c_{2} T^{2}+c_{1} T+c_{0} .
\end{aligned}
$$

The values of the coefficients $a_{i}$ for the dynamic viscosity, $b_{i}$ for the surface tension and $c_{i}$ for Henry's constant $(i=0-3)$ are given in Table 1 . The correlations for the temperature dependence of the properties of water were summarized in previous work $^{14}$.

Figure 1(a) compares the dissolution behavior of a bubble in two alkanes and water for a constant temperature $T=300 \mathrm{~K}$. The temporal evolution of the bubble radius, $R(t)$, is obtained by solving numerically equation 5 in combination with equations 6-9 for decane and hexadecane, and the corresponding equations given in ref. 14 for water. A comparison of decane and hexadecane highlights the effect of alkane chain length. Dissolution is faster in decane than in hexadecane. The solubility of nitrogen decreases with increasing alkane chain length ${ }^{25}$. The solubility of nitrogen at $300 \mathrm{~K}$ and ambient pressure in hexadecane $\left(k_{H} \approx 4 \times 10^{-5} \mathrm{~mol} \mathrm{~m}^{-3} \mathrm{~Pa}^{-1}\right)$ is lower than in decane $\left(k_{H} \approx 6 \times 10^{-5} \mathrm{~mol} \mathrm{~m}^{-3} \mathrm{~Pa}^{-1}\right)$, hence dissolution is faster in decane. In addition, the mass 
diffusivity of nitorgen in liquid $n$-alkanes $\left(\mathrm{C}_{6}-\mathrm{C}_{16}\right)$ at ambient conditions generally decreases with increasing chain length ${ }^{26}$, again leading to faster dissolution in decane than hexadecane. It is also clearly seen that bubble dissolution in water at constant temperature is much slower than in alkanes, primarily because the solubility of nitrogen in water is one order of magnitude lower than in the two alkanes $\left(k_{H} \approx 6 \times 10^{-6} \mathrm{~mol} \mathrm{~m}^{-3} \mathrm{~Pa}^{-1}\right)$.

Table 1. Physicochemical properties of alkanes, taken from refs. 19, 20, 21, 22, 23, 24.

\begin{tabular}{|l|c|c|c|c|}
\hline & $\boldsymbol{a}_{\mathbf{3}}\left(\mathrm{Pa} \mathrm{s} \mathrm{K}^{-3}\right)$ & $\boldsymbol{a}_{\mathbf{2}}\left(\mathrm{Pa} \mathrm{s} \mathrm{K}^{-2}\right)$ & $\boldsymbol{a}_{\mathbf{1}}\left(\mathrm{Pa} \mathrm{s} \mathrm{K}^{-1}\right)$ & $\boldsymbol{a}_{\mathbf{0}}(\mathrm{Pa} \mathrm{s})$ \\
\hline Hexadecane $^{19}$ & $-1.014 \times 10^{-8}$ & $1.021 \times 10^{-5}$ & $-3.453 \times 10^{-3}$ & $3.940 \times 10^{-1}$ \\
\hline Decane $^{20}$ & $-8.712 \times 10^{-10}$ & $9.285 \times 10^{-7}$ & $-3.346 \times 10^{-4}$ & $4.118 \times 10^{-2}$ \\
\hline & $\boldsymbol{b}_{\mathbf{3}}\left(\mathrm{N} \mathrm{m}^{-1} \mathrm{~K}^{-3}\right)$ & $\boldsymbol{b}_{\mathbf{2}}\left(\mathrm{N} \mathrm{m}^{-1} \mathrm{~K}^{-2}\right)$ & $\boldsymbol{b}_{\mathbf{1}}\left(\mathrm{N} \mathrm{m}^{-1} \mathrm{~K}^{-1}\right)$ & $\boldsymbol{b}_{\mathbf{0}}\left(\mathrm{N} \mathrm{m}^{-1}\right)$ \\
\hline Hexadecane $^{21}$ & 0 & 0 & $-8.540 \times 10^{-5}$ & $5.251 \times 10^{-2}$ \\
\hline Decane $^{22}$ & $-9.259 \times 10^{-9}$ & $8.998 \times 10^{-6}$ & $-3.004 \times 10^{-3}$ & $3.649 \times 10^{-1}$ \\
\hline & $\boldsymbol{c}_{\mathbf{3}}\left(\mathrm{mol} \mathrm{m}^{-3} \mathrm{~Pa}^{-1} \mathrm{~K}^{-3}\right)$ & $\boldsymbol{c}_{\mathbf{2}}\left(\mathrm{mol} \mathrm{m}^{-3} \mathrm{~Pa}^{-1} \mathrm{~K}^{-2}\right)$ & $\boldsymbol{c}_{\mathbf{1}}\left(\mathrm{mol} \mathrm{m}^{-3} \mathrm{~Pa}^{-1} \mathrm{~K}^{-1}\right)$ & $\boldsymbol{c}_{\mathbf{0}}\left(\mathrm{mol} \mathrm{m}^{-3} \mathrm{~Pa}^{-1}\right)$ \\
\hline Hexadecane $^{23}$ & $-1.258 \times 10^{-12}$ & $1.601 \times 10^{-9}$ & $-5.371 \times 10^{-7}$ & $9.354 \times 10^{-5}$ \\
\hline Decane $^{24}$ & 0 & $6.921 \times 10^{-10}$ & $-3.087 \times 10^{-7}$ & $9.526 \times 10^{-5}$ \\
\hline
\end{tabular}

Figure 1(b) shows the temperature dependence of Henry's constants for water, decane and hexadecane, normalized by the value at $300 \mathrm{~K}, k_{H 0}=k_{H}(T=300 \mathrm{~K})$. Interestingly, Henry's constant for alkanes shows the opposite trend with changing temperature than for water, that is, the solubility of nitrogen in water decreases with increasing temperature, whereas it increases with increasing temperature for decane and hexadecane. In contrast, surface tension and viscosity have the same qualitative behavior for all three solvents (not shown).

The effect on bubble dissolution of the change in solubility with temperature is shown in Figure 1(c). The bubbles are subject to a heating rate $\dot{T}=5 \mathrm{~K} \mathrm{~min}^{-1}$ from an initial temperature $T_{0}=$ $300 \mathrm{~K}$ to a final temperature $T_{f}=320 \mathrm{~K}$. Because of the opposite trend of $k_{H}(T)$ in water and alkanes, the model predicts that the bubble will grow in water and dissolve in decane or hexadecane. The predicted behavior for water is consistent with previous experimental observations that bubble dissolution in water is enhanced by cooling ${ }^{14}$, because cooling increases the gas solubility and hence induces under-saturation. Conversely, heating decreases the gas solubility and induces supersaturation, leading to bubble growth in water. In alkanes, dissolution is faster compared with the case at constant temperature of Figure 1(a). The prediction for decane and hexadecane is qualitatively compared with experimental results in the Results and Discussion section. The predictions of the model cannot be compared quantitatively with the results due to the geometry of the experiment. The 
model assumes an unbounded fluid, while in the experiment the bubbles are in contact with the wall of the sample cell due to buoyancy, and confinement effects are known to slow down dissolution ${ }^{16}$.

(a)

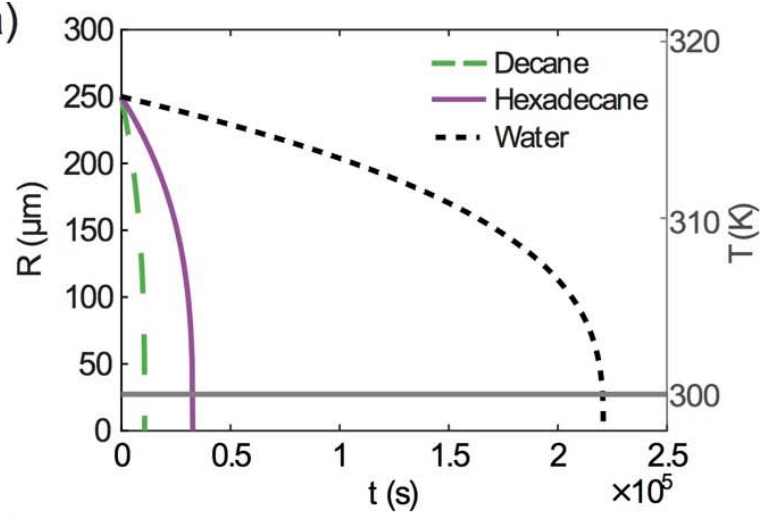

(c)

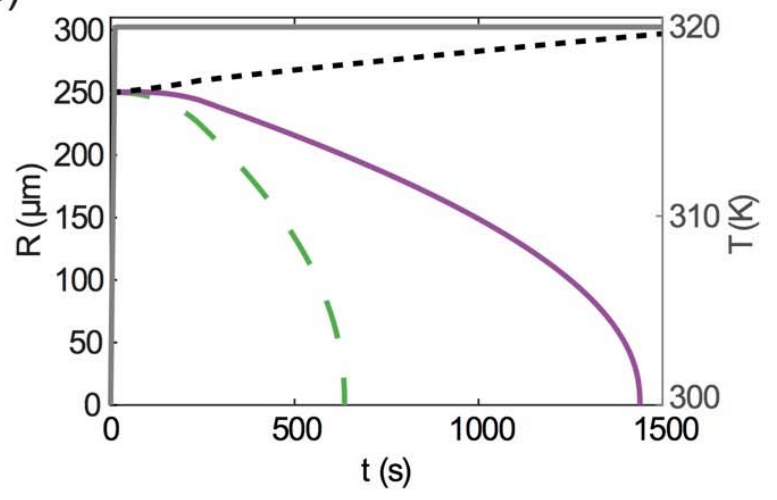

(b)

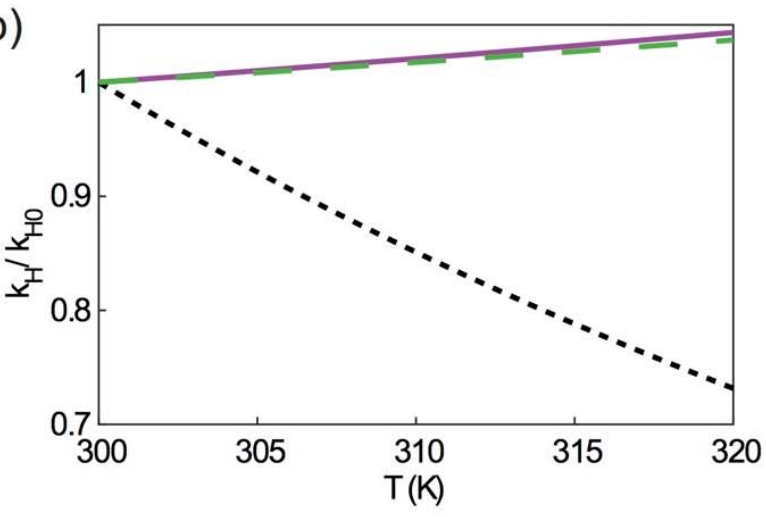

(d)

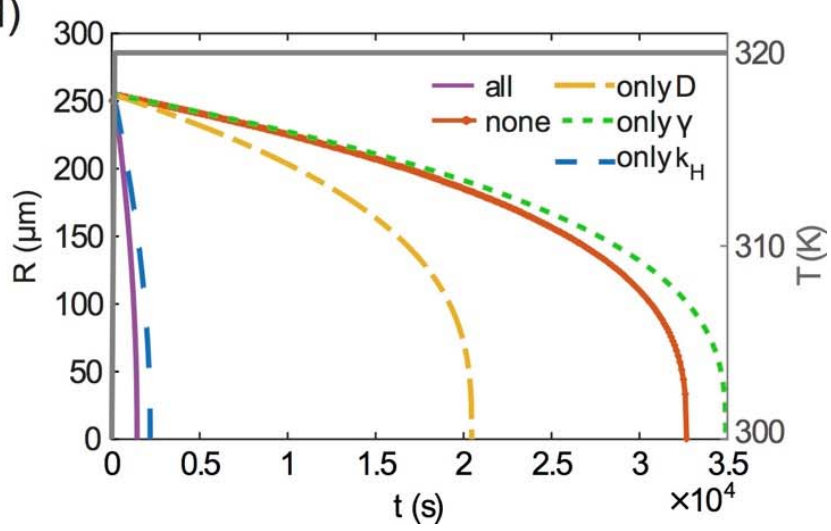

Figure 1. Model predictions for bubble dissolution in alkanes. (a) Temporal evolution of the radius of a bubble with initial radius $R_{0}=250 \mu \mathrm{m}$ in decane, hexadecane and water at constant temperature $T=300 \mathrm{~K}$. (b) Relative change in Henry's constant with temperature for decane, hexadecane and water over the temperature range $T=300-320 \mathrm{~K} . k_{\mathrm{H} 0}$ is the Henry's constant at $300 \mathrm{~K}$. (c) Temporal evolution of the radius of a bubble with initial radius $R_{0}=250 \mu \mathrm{m}$ in decane, hexadecane and water upon heating from $300 \mathrm{~K}$ to $320 \mathrm{~K}$ at a rate $\dot{T}=5 \mathrm{~K} \mathrm{~min}^{-1}$. (d) Effect of temperature-dependence of each physicochemical property of hexadecane, while keeping other properties constant: Henry's constant (blue), mass diffusivity (yellow), and surface tension (green). Dissolution behavior when all properties are temperature dependent (purple), and all properties are constant (orange) are shown for comparison.

To confirm the hypothesis that enhanced bubble dissolution in alkanes upon heating is caused by the increase in gas solubility, equation 5 can be solved allowing only one physicochemical property to vary with temperature, while the others remain constant. Figure 1(d) shows the results of this comparison for hexadecane. It can be seen that, with increasing temperature, both the increase in Henry's constant, $k_{H}$, and the increase in mass diffusivity, $D$, lead to enhanced bubble dissolution. On the other hand, the surface tension $\gamma$ decreases and hence the Laplace pressure is reduced, resulting 
in slower dissolution. A comparison with the prediction that includes the temperature dependence of all parameters suggests that the increase in gas solubility with temperature has the largest influence on enhancing bubble dissolution.

We also checked the effect of an elevated, constant temperature $T_{0}=320 \mathrm{~K}$ on bubble dissolution in alkanes (not shown). Because of the higher gas solubility, bubble dissolution is faster at a constant temperature $T_{0}=320 \mathrm{~K}$ than at a constant temperature $T_{0}=300 \mathrm{~K}$, assuming a saturation $f=1$ in both cases. However, the case of an increase in temperature is the one that leads to the fastest dissolution, because in this case the saturation decreases to $f<1$. When the temperature of an initially saturated solution of hexadecane $(f=1)$ at $T_{0}=300 \mathrm{~K}$ is increased by $\Delta T=+20 \mathrm{~K}$, the saturation ratio becomes $f \approx 0.95$.

\section{Experimental Section}

Particle-stabilized bubbles in alkanes. Fluorinated platelet sericite clay particles (PF-12) were used to stabilize air bubbles in alkanes ${ }^{12}$. The particles are formed from hydrophilic sericite (Daito Kasei, Japan) coated with a fluorinating agent (perfluoroalkyl phosphate diethanolamine salt), making the particles oleophobic. The platelets have an average lateral size of around $5 \mu \mathrm{m}^{12}$. Decane (SigmaAldrich, $\geq 99 \%$ ) and hexadecane (Sigma-Aldrich, 99\%) were used as received. The alkanes were initially equilibrated at atmospheric pressure to obtain a saturation $f \approx 1$. The desired concentration of particles for bubble preparation was calculated as percentage weight of particles per volume of solvent $(\% \mathrm{w} / \mathrm{v})$. Vigorous shaking with a vortex mixer was used to generate the bubbles. A small number of bubbles was transferred into an observation chamber made of a microscope glass slide and a coverslip, separated by a spacer, and filled with clean alkane.

Experimental setup. The observation chamber was placed on a temperature-controlled stage (THMS600, Linkam). The rate of change of temperature and the final temperature were set using the provided software Linksys32. Images were recorded in reflection mode every $10 \mathrm{~s}$ with a CMOS camera (Thorlabs) mounted on an upright microscope (BXFM, Olympus) with $10 \times$ magnification. The thermal time constant $\tau_{c}=L_{\text {th }}^{2} / \alpha$, where $\alpha$ is the thermal diffusivity and $L_{\text {th }}$ the thermal penetration length, was used to estimate the time delay in heat transfer from the heating plate to the sample. The thermal diffusivity at ambient conditions of glass ${ }^{27}$ and hexadecane ${ }^{28}$ are similar, and can be approximated by $\alpha \approx 10^{-7} \mathrm{~m}^{2} \mathrm{~s}^{-1}$. The thermal diffusion length is taken to be the thickness of the glass slide that separates the heat source from the solution, $L \approx 1 \mathrm{~mm}$. The thermal time 
constant is therefore $\tau_{c} \approx 10 \mathrm{~s}$. Due to the comparatively long timescales of the experiments $(>$ $10^{3} \mathrm{~s}$ ), the effects of transient heat transfer can be considered to be negligible.

Image analysis. The radius of uncoated, spherical bubbles was tracked using image analysis routines in Matlab (MathWorks, Natick, MA, USA). The function edge with the Sobel method was used to identify the pixels on the edge of the bubble. The measured area $A$ of the circle enclosed by the edge was then converted into bubble radius $R$. For particle-stabilized bubbles, the shape during dissolution can deviate significantly from spherical. In this case, the temporal evolution of the bubble size was quantified only where deviations from spherical shape remained reasonably small. The edge was smoothened by first dilating (imdilate) and then thinning (bwmorph). The edges of small objects, such as impurities, were then filtered out using the function bwareaopen. The area $A$ enclosed by the edge was converted into an effective radius $R$, that is, the radius of a circle with the same area $A$.

\section{Results and Discussion}

We first discuss the dissolution behavior of uncoated air bubbles in either decane or hexadecane at constant temperature and following an increase in temperature. This is followed by a discussion of the stability of particle-coated bubbles at constant temperature and following changes in temperature, including complex temperature cycles.

Effect of alkane chain length on dissolution rate of uncoated bubbles. First we compare the dissolution behavior of uncoated bubbles in decane and hexadecane at constant temperature $T=$ $300 \mathrm{~K}$ in saturation conditions, $f \approx 1$. Figure $2(\mathrm{a})$ shows the temporal evolution of the radius of air bubbles in the two alkanes. The initial bubble radii are $R_{0} \approx 130 \mu \mathrm{m}$ in decane and $R_{0} \approx 160 \mu \mathrm{m}$ in hexadecane, respectively. The dissolution time in decane, $t_{\mathrm{d}} \approx 2,880 \mathrm{~s}$ (48 mins), was significantly shorter than in hexadecane, $t_{\mathrm{d}} \approx 18,000 \mathrm{~s}(5 \mathrm{hrs})$. This experimental observation agrees qualitatively with the prediction of the model (see Figure 1(a)): dissolution is slower in hexadecane than in decane, because of the decrease in gas solubility and mass diffusivity with increasing alkane chain length.

Enhanced dissolution of uncoated bubbles in alkanes upon heating. The effect of increasing temperature on the dissolution of uncoated bubbles in decane is discussed first. Figure 2(b) shows the time evolution of the radii of two uncoated bubbles, one at a constant temperature $T=300 \mathrm{~K}$, and 
the other with a rapid increase in temperature from $T=300 \mathrm{~K}$ to $T=320 \mathrm{~K}$ at a heating rate $\dot{T}=$ $5 \mathrm{~K} / \mathrm{min}$. The initial radii of the two bubbles are $R_{0} \approx 140 \mu \mathrm{m}$.

(a)

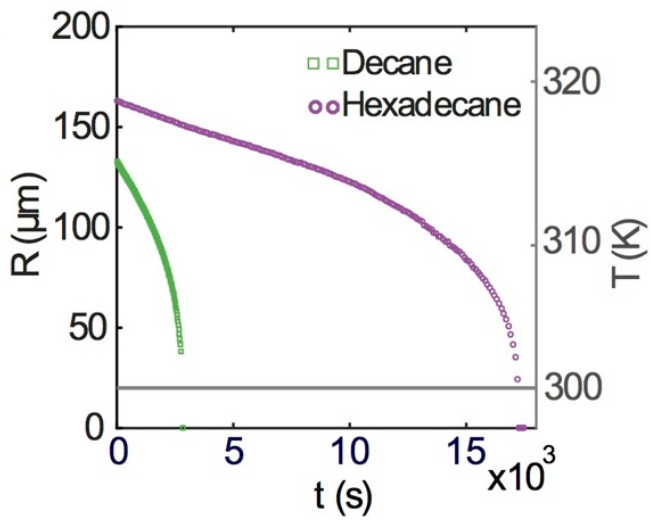

(b)

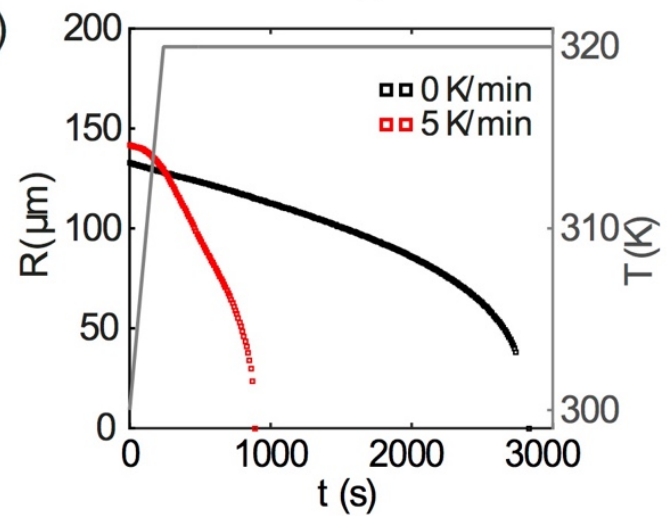

(c)

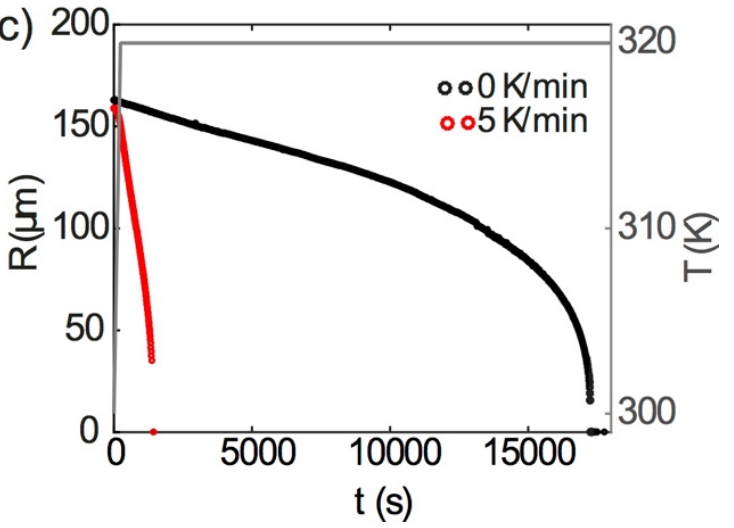

Figure 2. Experimental results on the dissolution behavior of uncoated bubbles in alkanes. (a) Effect of alkane chain length on dissolution behavior at constant temperature $T=300 \mathrm{~K}$. (b) Effect of heating $\left(\dot{T}=5 \mathrm{~K} \mathrm{~min}^{-1}\right)$ on dissolution behavior in decane. (c) Effect of heating $\left(\dot{T}=5 \mathrm{~K} \mathrm{~min}^{-1}\right)$ on dissolution behavior in hexadecane.

It can be seen that the dissolution rate is significantly enhanced by increasing the temperature. The dissolution times are $t_{\mathrm{d}} \approx 2,800 \mathrm{~s}(47 \mathrm{~min})$ and $t_{\mathrm{d}} \approx 900 \mathrm{~s}(15 \mathrm{~min})$ for constant temperature and upon heating, respectively. Similar results are obtained for hexadecane, as shown in Figure 2(c). The initial radii of the two bubbles are $R_{0} \approx 160 \mu \mathrm{m}$, and the dissolution times are $t_{\mathrm{d}} \approx 17,000 \mathrm{~s}(5 \mathrm{hr}$ ) 
and $t_{\mathrm{d}} \approx 1,400 \mathrm{~s}(23 \mathrm{~min})$ for constant temperature and upon heating, respectively. The results for decane and hexadecane are in qualitative agreement with the prediction that increasing the temperature speeds up bubble dissolution in alkanes (see Figure 1(c)).

(a)

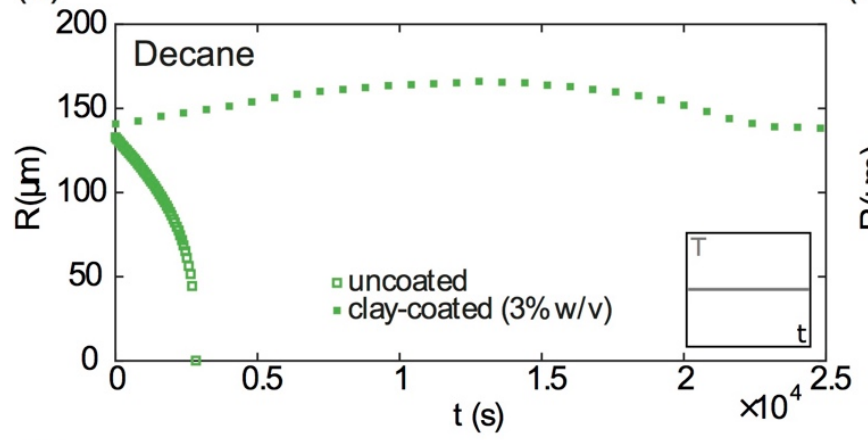

(c)

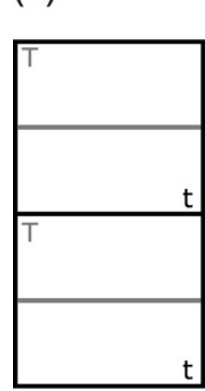

(b)

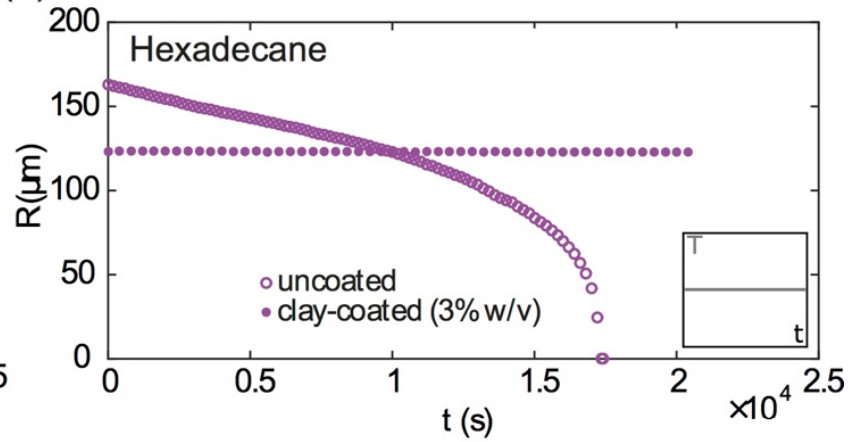

(d)
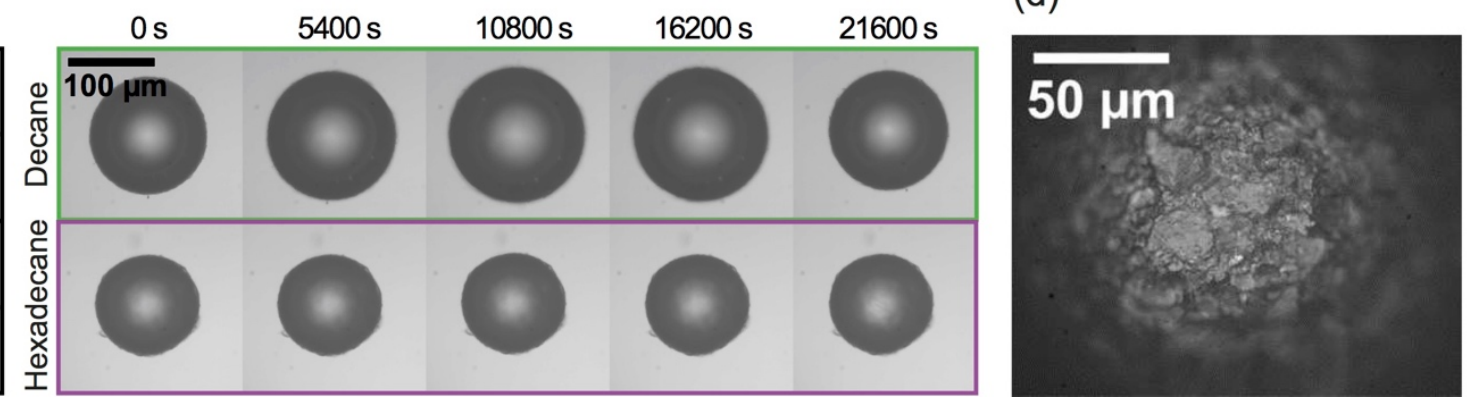

Figure 3. Stability of clay-coated bubbles in alkanes at constant temperature $T=300 \mathrm{~K}$. Clay-coated bubbles are prepared from $3 \% \mathrm{w} / \mathrm{v}$ clay dispersions in alkanes. (a) Temporal evolution of the radius of uncoated (open symbols) and clay-coated bubbles (filled symbols) in decane. (b) Temporal evolution of the radius of uncoated (open symbols) and clay-coated bubbles (filled symbols) in hexadecane. (c) Image sequence of clay-coated bubbles in decane (top) and hexadecane (bottom). (d) Close-up of a clay-coated bubble in hexadecane after approximately $900 \mathrm{~s}(10 \mathrm{~min})$.

Long-term stability of particle-coated bubbles in alkanes at constant temperature. In contrast to uncoated bubbles, clay particle-coated bubbles are found to be stable at constant temperature $T=$ $300 \mathrm{~K}$ in decane and hexadecane. Figures $3(\mathrm{a}-\mathrm{b})$ show a comparison of the time evolution of the radius of uncoated and clay-coated bubbles in the two alkanes. The particle-coated bubbles are prepared from a $3 \% \mathrm{w} / \mathrm{v}$ clay dispersion in alkane and have initial radii $R_{0} \approx 140 \mu \mathrm{m}$ and $R_{0} \approx$ $120 \mu \mathrm{m}$ in decane and hexadecane, respectively, similar to the initial radii of the uncoated bubbles. It can be seen that there is no observable reduction in radius of clay-coated bubbles in decane over a time $t=25,000 \mathrm{~s}$ ( $\sim 7 \mathrm{hrs}$ ) (Figure 3(a)) or in hexadecane over a time $t=21,600 \mathrm{~s} \mathrm{(6} \mathrm{hrs)} \mathrm{(Figure}$ 3(b)). The uncoated bubbles have dissolved completely over these timescales. During the experiment, 
the radius of the clay-coated bubble in decane underwent a fluctuation of approximately $10 \%$, possibly due to a change in temperature, but the bubble did not dissolve. This behavior is in line with the remarkable long-term stability of air-in-oil foams stabilized by the same oleophobic particles ${ }^{12}$. The morphology of the clay-coated bubbles in both alkanes also remained unchanged, as shown by the image sequences in Figure 3(c). A close examination of a single bubble (Figure 3(d)) revealed that the clay particles form a densely packed layer on the bubble surface with very high surface coverage. It was not possible to quantify the surface coverage from the optical microscopy images however. The stability imparted by the clay particles can be attributed to a reduction in the gas-liquid interfacial area available for diffusion ${ }^{29}$, a reduction in Laplace pressure ${ }^{30,31}$, the elasticity of the particle monolayer ${ }^{32}$, or a combination of these effects.

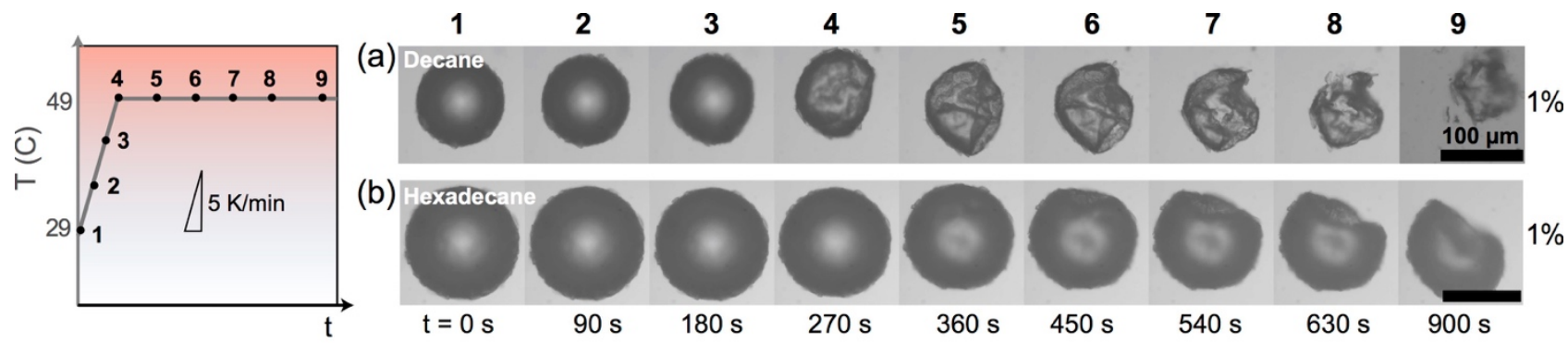

(c)
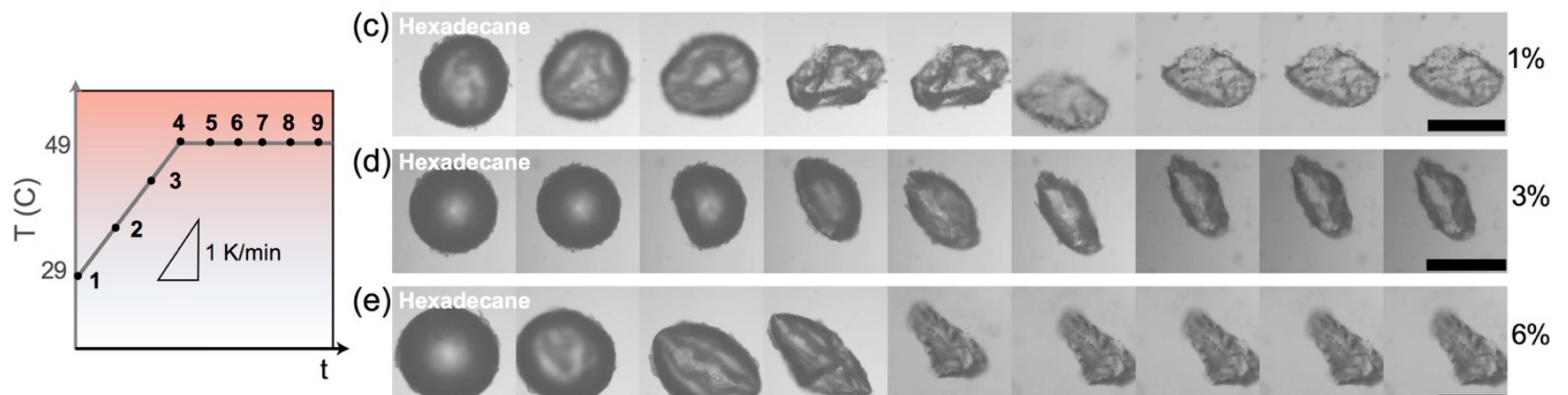

(d)

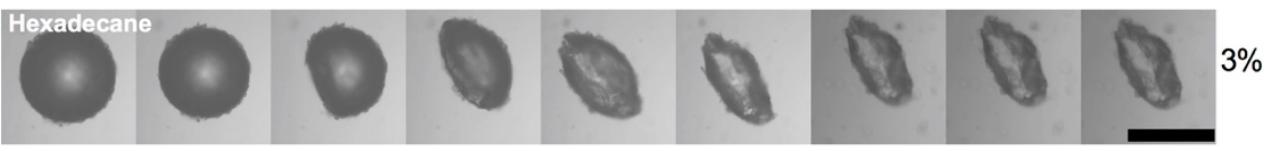

(e)

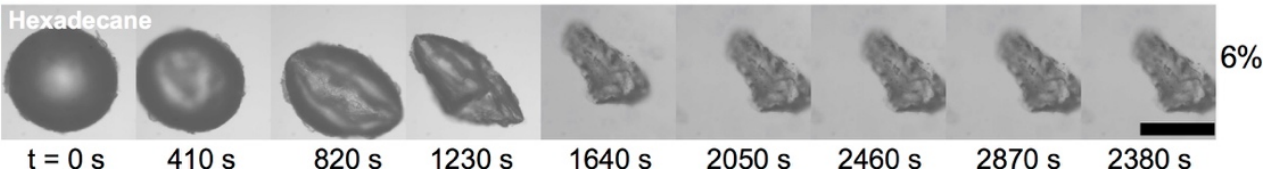

Figure 4. Dissolution behavior of clay-coated bubbles upon heating $\left(T_{0}=300 \mathrm{~K}, \Delta T=20 \mathrm{~K}\right)$. (a) $1 \% \mathrm{w} / \mathrm{v}$ clay in decane, $\dot{T}=5 \mathrm{~K} \mathrm{~min}^{-1}$. (b) $1 \% \mathrm{w} / \mathrm{v}$ clay in hexadecane, $\dot{T}=5 \mathrm{~K} \mathrm{~min}^{-1}$. (c) $1 \% \mathrm{w} / \mathrm{v}$ clay in hexadecane, $\dot{T}=1 \mathrm{~K} \mathrm{~min}^{-1}$. (d) $3 \% \mathrm{w} / \mathrm{v}$ clay in hexadecane, $\dot{T}=1 \mathrm{~K} \mathrm{~min}^{-1}$. (e) $6 \% \mathrm{w} / \mathrm{v}$ clay in hexadecane, $\dot{T}=1 \mathrm{~K} \mathrm{~min}^{-1}$. All scale bars $100 \mu \mathrm{m}$.

Stability of clay-coated bubbles in alkanes upon heating. We explored the effect of heating on the stability of clay-coated bubbles. We compared the behavior of bubbles in decane and in hexadecane, the effect of surface coverage by clay particles and the effect of the heating rate. Surface coverage was controlled indirectly through the bulk concentration of clay in the alkane dispersion used to prepare the bubbles but could not be quantified directly. Figures 4(a-b) show image sequences of 
clay-coated bubbles made from 1\% w/v dispersions in decane (Figure 4(a)) and hexadecane (Figure 4(b)) respectively, and heated at a rate $\dot{T}=5 \mathrm{~K} \mathrm{~min}^{-1}$ from an initial temperature $T_{0}=300 \mathrm{~K}$ with $\Delta T=20 \mathrm{~K}$. Gas diffuses out of the bubbles leading to a decrease in volume. The layer of particles behaves as a solid-like film and crumples upon area compression, without any apparent shedding of particles, in contrast to latex-stabilized air bubbles dissolving in water upon cooling ${ }^{14}$ or undergoing compression by other mechanisms ${ }^{33,34}$. We checked the effect of the rate of area compression on this mechanical instability of the particle layer by changing the heating rate. Figure 4(c) shows an image sequence of a bubble made from a $1 \% \mathrm{w} / \mathrm{v}$ dispersion in hexadecane heated at a rate $\dot{T}=1 \mathrm{~K} \mathrm{~min}^{-1}$ (initial temperature $T_{0}=300 \mathrm{~K}, \Delta T=20 \mathrm{~K}$ ). The dissolution behavior is similar to the one observed for $\dot{T}=5 \mathrm{~K} \mathrm{~min}^{-1}$ (Figure 4(b)).

Next we consider the effect of surface coverage by particles. Figures $4(\mathrm{~d}-\mathrm{e})$ show image sequences of clay-coated bubbles made from a 3\% w/v (Figure 4(d)) and a 6\% w/v (Figure 4(e)) dispersion in hexadecane, respectively, and heated at a rate $\dot{T}=1 \mathrm{~K} \mathrm{~min}^{-1}$ (initial temperature $T_{0}=$ $300 \mathrm{~K}, \Delta T=20 \mathrm{~K})$. A comparison of Figures $4(\mathrm{c}-\mathrm{e})$ suggests that the bubbles dissolve completely below a certain coverage by particles (bubbles made from $1 \% \mathrm{w} / \mathrm{v}$ ), while for sufficiently high surface coverage (bubbles made from $3 \% \mathrm{w} / \mathrm{v}$ and $6 \% \mathrm{w} / \mathrm{v}$ dispersion) small gas pockets appear to remain trapped in the crumpled shell of particles and resists further dissolution.

To confirm the above hypothesis, we subjected the system to a heating-cooling cycle for three different surface coverages. Figure 5 shows the morphological evolution of bubbles prepared from 1\% w/v (Figure 5(a)), 3\% w/v (Figure 5(b)), and 6\% w/v dispersions in hexadecane (Figure 5(c)). Initially the bubbles were briefly subjected to cooling below ambient temperature $\left(T_{0}=\right.$ $302 \mathrm{~K}, T_{\text {cold }}=290 \mathrm{~K}, \Delta T=-10 \mathrm{~K}$ ) to cause them to expand slightly and start the experiment from a spherical shape. The system was then heated $\left(\Delta T=+30 \mathrm{~K}\right.$, i.e. $\left.T_{\text {hot }}=T_{0}+20 \mathrm{~K}\right)$, after which the temperature was held constant for 8 hours and the dynamics of bubble dissolution was observed. The bubbles remained spherical until the temperature reached ambient temperature. As the temperature was increased further, the saturation ratio decreased to $f<1$ and the bubbles started to dissolve, leading to buckling and crumpling of the particle layer. After 8 hours, the system was cooled back to ambient temperature $T_{0}=302 \mathrm{~K}(\Delta T=-20 \mathrm{~K})$ to check for any gas retention within the bubble, as bubbles are expected to grow upon cooling. As can be seen in Figures 5(b-c), gas was retained within the crumpled clay shell for higher surface coverage (corresponding to $3 \% \mathrm{w} / \mathrm{v}$ and $6 \% \mathrm{w} / \mathrm{v}$ clay in dispersion). No re-expansion was observed for lower surface coverage ( $1 \% \mathrm{w} / \mathrm{v}$ clay in dispersion) indicating the absence of any retained gas at the end of the heating stage. The local retention of gas can be ascribed to a non-uniform surface coverage by particles, with the threshold in surface coverage 
for gas retention being overcome only for sufficiently large initial coverage. Hydrophobic clay platelets can also form multilayers due to their cohesive (van der Waals) interactions, possibly creating a layer that is impermeable to gas diffusion.

(a)
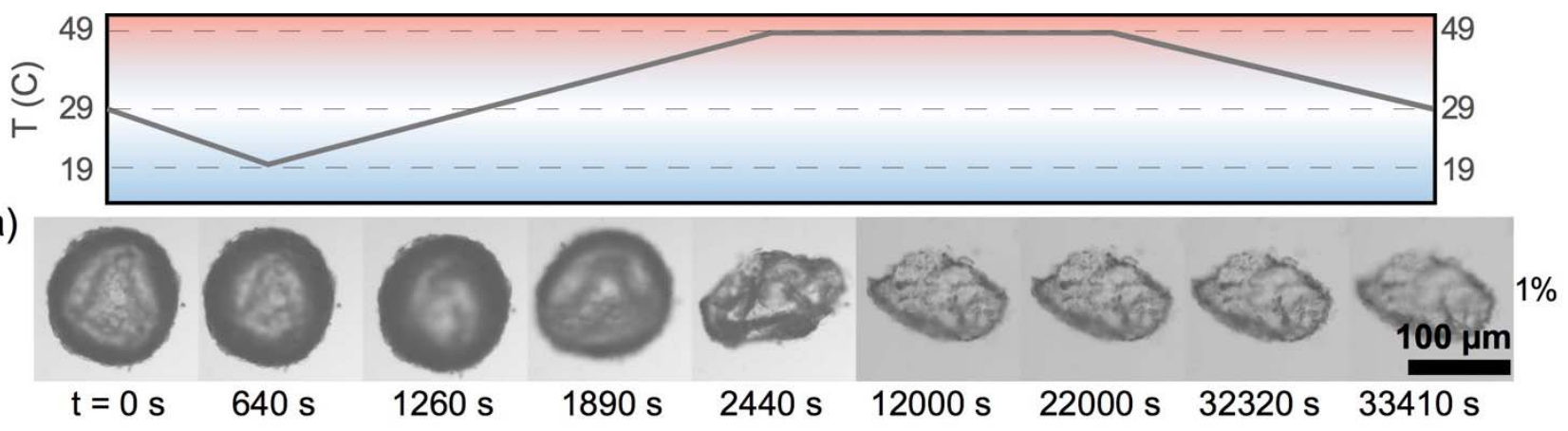

(b)

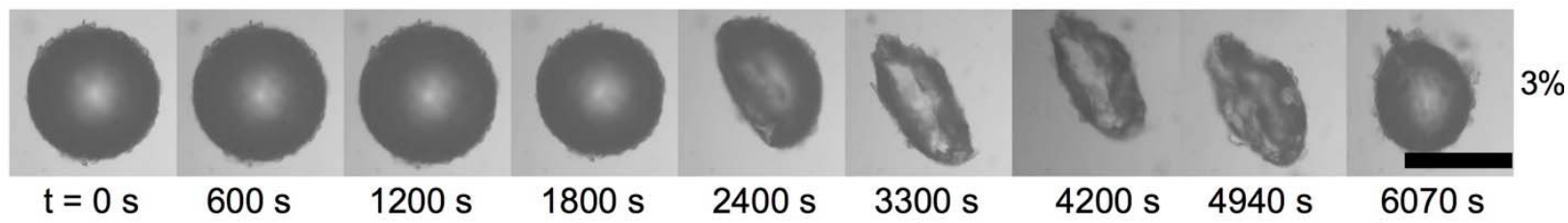

(c)

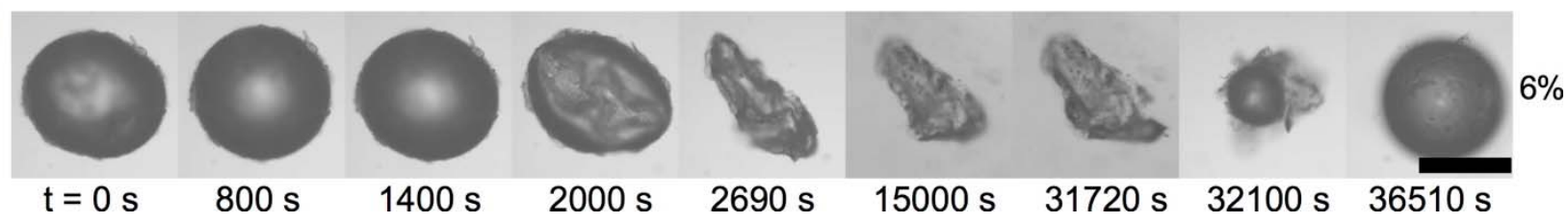

Figure 5. Effect of cooling back to room temperature after heating. Clay-coated bubbles in hexadecane are initially cooled for $600 \mathrm{~s}(\Delta T=-10 \mathrm{~K})$, then the temperature is increased above room temperature $(\Delta T=$ $30 \mathrm{~K}$ ) and held constant for $8 \mathrm{hr}$ (up to 31,000 s) and finally the bubbles are cooled back to room temperature

(above $31200 \mathrm{~s}$ ). (a) For 1\% w/v clay, the bubble is destroyed. For (b) 3\% w/v clay and (c) $6 \% \mathrm{w} / \mathrm{v}$ clay, a gas pocket remains trapped in the particle shell and nucleates a bubble upon cooling back to room temperature. All scale bars $100 \mu \mathrm{m}$.

Effect of temperature cycling on stability of clay-coated bubbles. We further investigated the stability of clay-coated bubbles upon periodic temperature cycling. We subjected bubbles prepared from a $1 \% \mathrm{w} / \mathrm{v}$ dispersion in hexadecane to temperature fluctuations of constant amplitude $\Delta T=$ $\pm 5 \mathrm{~K}$ around ambient temperature $T_{0}=297 \mathrm{~K}$. Figure $6(\mathrm{a})$ shows the morphology of a bubble with an initial radius $R_{0} \approx 90 \mu \mathrm{m}$ at ambient temperature when it is heated and cooled for three cycles. The bubble undergoes growth and dissolution cycles as the temperature fluctuates. The bubble surface deforms upon heating but recovers its spherical shape upon cooling. The deviation from spherical shape is sufficiently small that an effective radius can be extracted from the projected area. Figures $6(b)$ and (c) show the effective bubble radius as a function of time and as a function of temperature, 
respectively. It can be seen that the periodic fluctuations in bubble radius are not in phase with the temperature fluctuations, and that the amplitude of the fluctuations decrease over time.

(a)

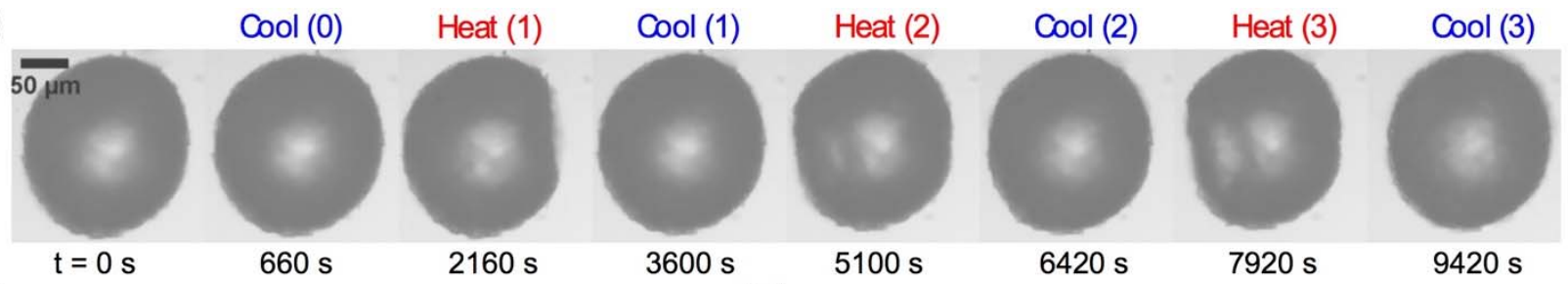

(b)

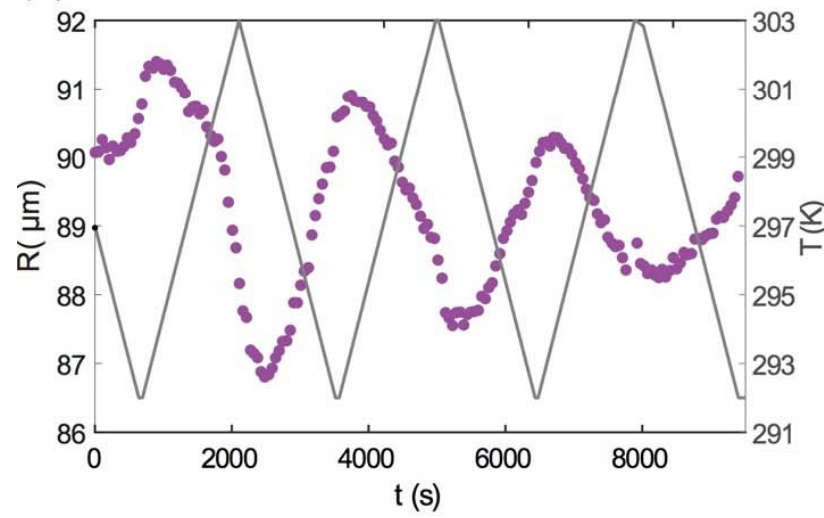

(c) 92

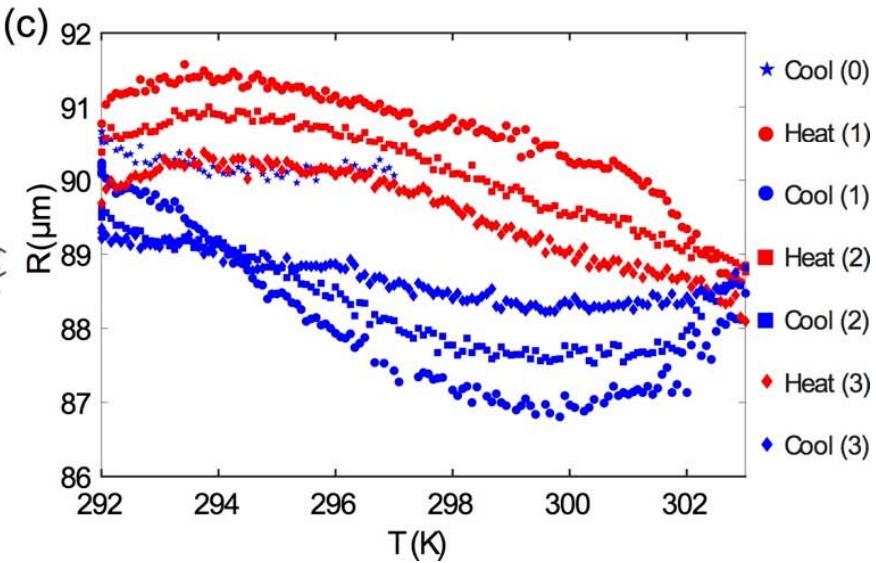

Figure 6. Clay-coated bubble (from $1 \% \mathrm{w} / \mathrm{v}$ dispersion) in hexadecane subjected to constant amplitude temperature oscillations between $T_{\text {cold }}=292 \mathrm{~K}$ and $T_{\text {hot }}=303 \mathrm{~K}$. (a) Image sequence of bubble morphology over 3 cycles of cooling and heating. (b) Temporal evolution of the bubble radius. (c) Bubble radius as a function of temperature.

One might expect the change from bubble dissolution to growth or from growth to dissolution (that is, the change in sign of $\dot{R}$ ), to occur for $f=1$. However, the rate of cooling, $\dot{T}$, changes from negative to positive half way through the time intervals during which $f<1$, and from positive to negative half way through the time intervals during which $f>1$. The interplay between the first term in equation (5), which depends on $f$, and the second term, which depends on $\dot{T}$, is likely to cause the observed phase difference between $R(t)$ and $T(t)$. The reduction in the amplitude of fluctuations of the radius may be attributed to changes in the morphology and mechanical properties of the particle layer upon repeated compression-expansion (see for instance ref. 32).

To gain further insight on the stability of clay-coated bubbles in alkanes under varying environmental conditions, the dissolution behavior of uncoated and coated bubbles was compared when subjected to more complex temperature cycling. To maintain a spherical shape of the coated bubbles throughout the experiment, the maximum temperature was set to a constant value $T_{\text {hot }}=T_{0}+1 \mathrm{~K}=300 \mathrm{~K}$, while the minimum temperature $T_{\text {cold }}$ was decreased in each cycle. In the first cycle, the temperature was first decreased to $T_{\text {cold }}=T_{0}-1 \mathrm{~K}$, and then increased back to $T_{\text {hot }}$ 
$(\Delta T=+2 \mathrm{~K})$. The temperature was then varied between $T_{\text {hot }}=300 \mathrm{~K}$ and $T_{\text {cold }}=T_{\text {hot }}-\Delta T$, with $\Delta T=3 \mathrm{~K}, 4 \mathrm{~K}, 5 \mathrm{~K}, 6 \mathrm{~K}$. All the heating/cooling rates are $\dot{T}= \pm 0.5 \mathrm{~K} \mathrm{~min}^{-1}$. The resulting temperature profile is shown in Figure 7. The temperature profile is designed so that the temperature is below $T_{0}=299 \mathrm{~K}$, and therefore $f>1$, for most of the time, which should minimize long-term bubble dissolution.

(a)

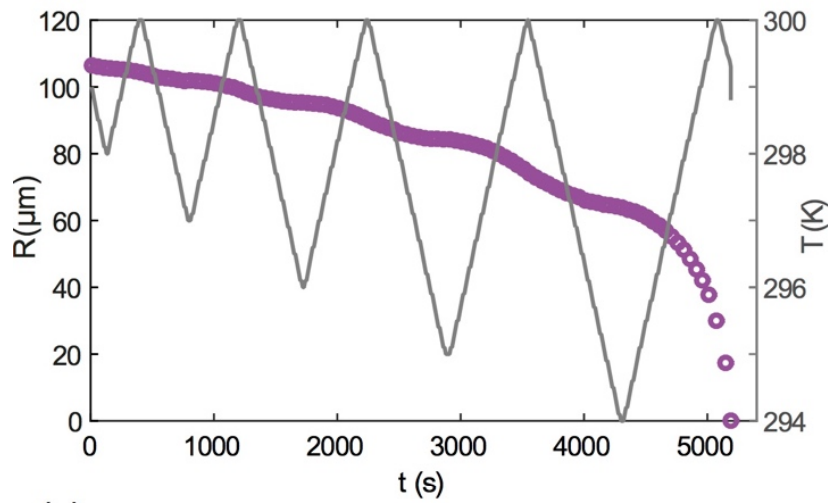

(c)

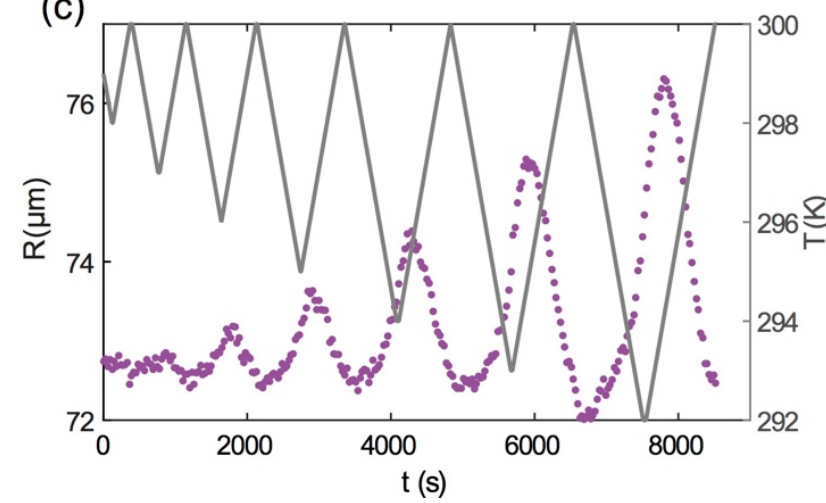

(b)

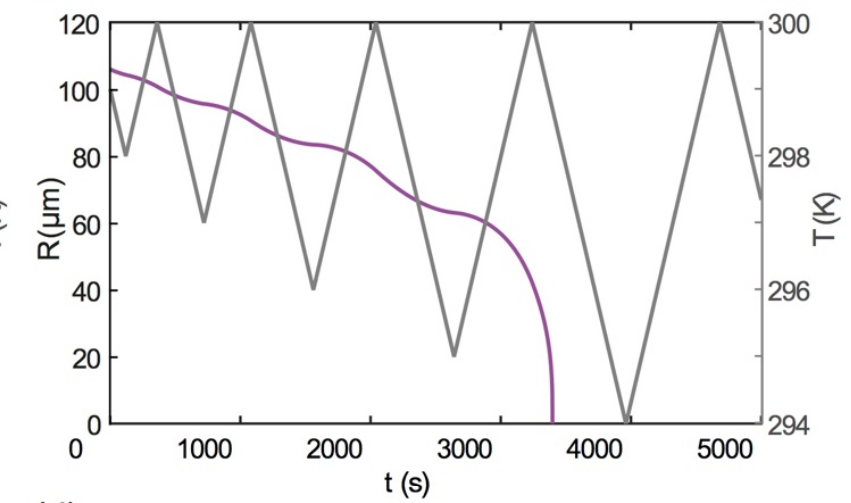

(d)

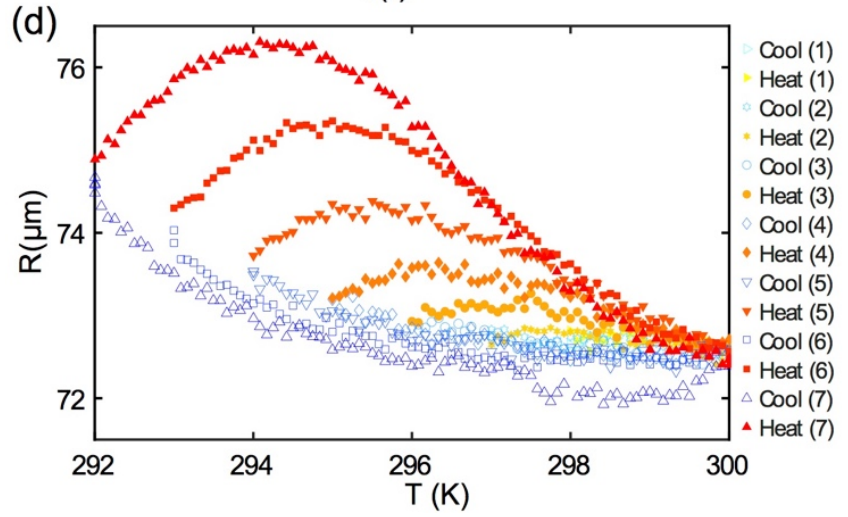

Figure 7. Behavior of uncoated and clay-coated bubbles in hexadecane subjected to temperature oscillations of increasing amplitude. (a) Experimental results for the time evolution of the radius of an uncoated bubble with initial radius $R_{0}=106 \mu \mathrm{m}$. (b) Predictions of the model for the time evolution of the radius of an uncoated bubble with initial radius $R_{0}=106 \mu \mathrm{m}$ and subjected to the same heating-cooling protocol as in experiment (other parameters given in Theory section). (c) Experimental results for the time evolution of the radius of a clay-coated bubble ( $1 \% \mathrm{w} / \mathrm{v})$ with initial radius $R_{0}=73 \mu \mathrm{m}$. (d) Experimental results for the radius of a clay-coated bubble as a function of temperature.

Figure 7(a) shows experimental results for the temporal evolution of the radius of an uncoated bubble in hexadecane with initial radius $R_{0} \approx 106 \mu \mathrm{m}$. At time $t=0$, the bubble does not grow, even though $f>1$ upon cooling, but continues to dissolve because the effect of the Laplace pressure dominates over that of super-saturation, i.e. $f<1+\frac{2 \gamma / R}{P_{0}}$. As the bubble radius decreases upon dissolution, the 
Laplace pressure continues to increase. Upon cooling in subsequent cycles, the effect of Laplace pressure increasingly dominates that of super-saturation, and hence the decrease in temperature only reduces the rate of dissolution, but does not halt it. The bubble eventually dissolves completely at $t_{\mathrm{d}} \approx 5,200 \mathrm{~s}$. Figure 7(b) shows the prediction obtained from the model, which is in qualitative agreement with the experimental results, although the predicted timescale for dissolution is shorter $\left(t_{\mathrm{d}} \approx 3,400 \mathrm{~s}\right)$ because of the effect of confinement in experiment ${ }^{16}$. The phase shift between $R(t)$ and $T(t)$ observed in Figure 6(b) is also observed in Figures 7(a-b). The fact that the phase shift is observed for uncoated bubbles confirms that it is not an effect of the particle layer. The fact that it is captured by the model suggests that it is not an effect of limitations in the experiment, but rather of the interplay between the first and second term in equation (5), which depend on $f-\frac{2 \gamma / R}{P_{0}}$ and $\dot{T}$, respectively, and which change sign at different times during the cycle. In contrast to an uncoated bubble, a clay-stabilized bubble prepared from a $1 \% \mathrm{w} / \mathrm{v}$ dispersion in hexadecane and subjected to the same temperature profile remained remarkably stable as shown in Figures 7(c-d), even though its initial radius $\left(R_{0} \approx 73 \mu \mathrm{m}\right)$ was smaller than that of the uncoated bubble. The bubble underwent periodic shrinkage and expansion cycles, similar to the case of constant amplitude reported in Figure 6 , with a slight increase in average radius. Because of the initially low dissolution rate, there is negligible reduction in bubble radius and the Laplace pressure remains relatively constant. Remarkably, the clay-particle coated bubble remains stable when subjected to a complex temperature profile for which an uncoated bubble dissolves completely.

\section{Summary and Conclusions}

We studied the stability of air bubbles in alkanes with or without a coating of fluorinated clay particles. Unlike uncoated bubbles, clay-coated bubbles are stable against dissolution at constant temperature, where dissolution is driven mainly by the Laplace pressure. The coating of particles can minimize the effect of the Laplace pressure through a reduction in the surface tension of the gasliquid interface, and through the elasticity of the particle monolayer. Investigations on the effect of temperature on the dissolution dynamics reveals that particle-coated bubbles are prone to dissolution and buckling when the temperature is increased. A model for bubble dissolution with time-dependent temperature suggests that the increase in Henry's constant, which leads to an under-saturated solution, is the governing factor for driving bubble instability. The coating of particles is therefore not sufficient to ensure stability against dissolution, because dissolution is not driven by the Laplace pressure but by under-saturation. When a clay-coated bubble is subjected to a step increase in 
temperature, the dense packing of the particle layer can temporarily slow down the dissolution rate in the short-term, but the bubble is prone to further buckling in the long-term. Particle shedding is not observed, likely because van der Waals attraction is dominant between the fluorinated clay platelets. By increasing the particle concentration, the packing density at the interface increases and this provides higher resistance to buckling even when exposed to an under-saturated oil phase. The stability of clay-coated bubbles is also retained when subjected to complex temperature cycles, during which the bubbles recover their spherical shape upon cooling. This work shows the potential for using such ultra-stable clay-coated bubbles in applications where foam stability in varying environmental conditions is a requirement.

\section{Acknowledgment}

This work was supported in part by the European Research Council, Starting Grant No. 639221 (V.G.).

\section{References}

1. Dickinson, E. Food emulsions and foams: Stabilization by particles. Current Opinion in Colloid \& Interface Science 2010, 15, 40-49.

2. Binks, B. P.; Davies, C. A.; Fletcher, P. D. I.; Sharp, E. L. Non-aqueous foams in lubricating oil systems. Colloids and Surfaces A: Physicochemical and Engineering Aspects 2010, 360, 198-204.

3. Hunter, T. N.; Pugh, R. J.; Franks, G. V.; Jameson, G. J. The role of particles in stabilising foams and emulsions. Advances in Colloid and Interface Science 2008, 137, 57-81.

4. Binks, B. P. Particles as surfactants - Similarities and differences. Current Opinion in Colloid \& Interface Science 2002, 7, 21-41.

5. Garbin, V. Colloidal particles: Surfactants with a difference. Physics Today 2013, 66, 68-69.

6. Tzoumaki, M. V.; Karefyllakis, D.; Moschakis, T.; Biliaderis, C. G.; Scholten, E. Aqueous foams stabilized by chitin nanocrystals. Soft Matter 2015, 11, 6245-6253.

7. Dickinson, E.; Ettelaie, R.; Kostakis, T.; Murray, B. S. Factors Controlling the Formation and Stability of Air Bubbles Stabilized by Partially Hydrophobic Silica Nanoparticles. Langmuir 2004, 20, 85178525.

8. Binks, B. P.; Horozov, T. S. Aqueous Foams Stabilized Solely by Silica Nanoparticles. Angewandte Chemie 2005, 117, 3788-3791. 
9. Murakami, R.; Bismarck, A. Particle-Stabilized Materials: Dry Oils and (Polymerized) Non-Aqueous Foams. Advanced Functional Materials 2010, 20, 732-737.

10. Binks, B. P.; Rocher, A.; Kirkland, M. Oil foams stabilised solely by particles. Soft Matter 2011, 7, 1800-1808.

11. Binks, B. P.; Tyowua, A. T. Influence of the degree of fluorination on the behaviour of silica particles at air-oil surfaces. Soft Matter 2013, 9, 834-845.

12. Binks, B. P.; Sekine, T.; Tyowua, A. T. Dry oil powders and oil foams stabilised by fluorinated clay platelet particles. Soft Matter 2014, 10, 578-589.

13. Sun, Q.; Li, Z.; Li, S.; Jiang, L.; Wang, J.; Wang, P. Utilization of Surfactant-Stabilized Foam for Enhanced Oil Recovery by Adding Nanoparticles. Energy \& Fuels 2014, 28, 2384-2394.

14. Poulichet, V.; Garbin, V. Cooling Particle-Coated Bubbles: Destabilization beyond Dissolution Arrest. Langmuir 2015, 31, 12035-12042.

15. Epstein, P. S.; Plesset, M. S. On the Stability of Gas Bubbles in Liquid-Gas Solutions. The Journal of Chemical Physics 1950, 18, 1505-1509.

16. Duncan, P. B.; Needham, D. Test of the Epstein-Plesset Model for Gas Microparticle Dissolution in Aqueous Media: Effect of Surface Tension and Gas Undersaturation in Solution. Langmuir 2004, 20, 2567-2578.

17. Lohse, D.; Zhang, X. Surface nanobubbles and nanodroplets. Reviews of Modern Physics 2015, 87, 9811035.

18. Krynicki, K.; Green, C. D.; Sawyer, D. W. Pressure and temperature dependence of self-diffusion in water. Faraday Discussions of the Chemical Society 1978, 66, 199-208.

19. Wohlfarth, C.; Wohlfahrt, B. C16. In Pure Organic Liquids, Lechner, M. D., Ed.; Springer Berlin Heidelberg, 2002, pp 724-757.

20. Wohlfarth, C.; Wohlfahrt, B. C10. In Pure Organic Liquids, Lechner, M. D., Ed.; Springer Berlin Heidelberg, 2002, pp 528-580.

21. Jasper, J. J.; Kring, E. V. The Isobaric Surface Tensions and Thermodynamic Properties of the Surfaces of a Series of n-Alkanes, C5 to C18, 1-Alkenes, C6 to C16, and of n-Decylcyclopentane, nDecylcyclohexane and n-Decylbenzene. The Journal of Physical Chemistry 1955, 59, 1019-1021.

22. Rolo, L. I.; Caço, A. I.; Queimada, A. J.; Marrucho, I. M.; Coutinho, J. A. P. Surface Tension of Heptane, Decane, Hexadecane, Eicosane, and Some of Their Binary Mixtures. Journal of Chemical \& Engineering Data 2002, 47, 1442-1445.

23. Tremper, K. K.; Prausnitz, J. M. Solubility of inorganic gases in high-boiling hydrocarbon solvents. Journal of Chemical \& Engineering Data 1976, 21, 295-299. 
24. Nasrifar, K.; Rahmanian, N. High-pressure solubility of light gases in heavy n-alkanes from a predictive equation of state: Incorporating Henry's law constant into binary interaction parameter. Fluid Phase Equilibria 2014, 381, 95-101.

25. Makranczy, J.; Megyery-Balog, K. M.; Rusz, L.; Patyi, L. Solubility of Gases in Normal-alkanes. Hungarian Journal of Industry and Chemistry 1976, 4, 269-280.

26. Winkelmann, J. Diffusion in Gases, Liquids and Electrolytes; Springer-Verlag Berlin Heidelberg; Vol. $15 \mathrm{~B} 1$.

27. Yang, G. Heat capacity and thermal diffusivity of a glass sample. Physical Review B 1992, 45, 157-160.

28. Vélez, C.; Khayet, M.; Ortiz de Zárate, J. M. Temperature-dependent thermal properties of solid/liquid phase change even-numbered n-alkanes: n-Hexadecane, n-octadecane and n-eicosane. Applied Energy 2015, 143, 383-394.

29. Azmin, M.; Mohamedi, G.; Edirisinghe, M.; Stride, E. P. Dissolution of coated microbubbles: The effect of nanoparticles and surfactant concentration. Materials Science and Engineering: C 2012, 32, $2654-$ 2658.

30. Kam, S. I.; Rossen, W. R. Anomalous Capillary Pressure, Stress, and Stability of Solids-Coated Bubbles. Journal of Colloid and Interface Science 1999, 213, 329-339.

31. Abkarian, M.; Subramaniam, A. B.; Kim, S.-H.; Larsen, R. J.; Yang, S.-M.; Stone, H. A. Dissolution Arrest and Stability of Particle-Covered Bubbles. Physical Review Letters 2007, 99, 188301.

32. Stocco, A.; Rio, E.; Binks, B. P.; Langevin, D. Aqueous foams stabilized solely by particles. Soft Matter 2011, 7, 1260-1267.

33. Poulichet, V.; Garbin, V. Ultrafast desorption of colloidal particles from fluid interfaces. Proceedings of the National Academy of Sciences 2015, 112, 5932-5937.

34. Poulichet, V.; Huerre, A.; Garbin, V. Shape oscillations of particle-coated bubbles and directional particle expulsion. Soft Matter 2017, 13, 125-133. 
Table of Contents entry

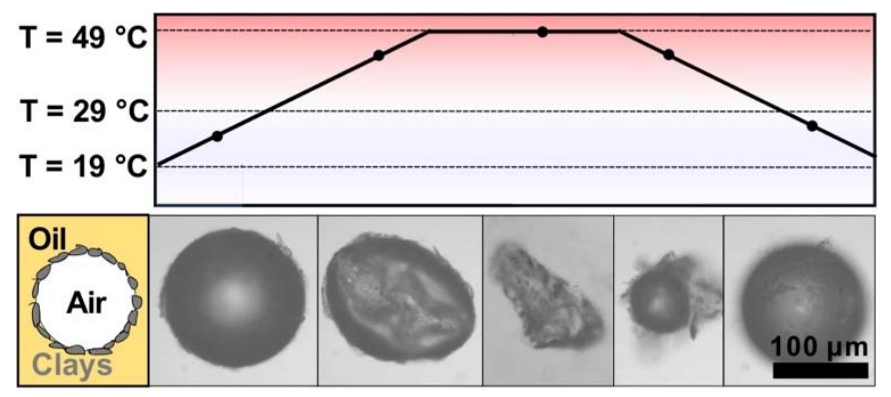

